\title{
Lessons of the month: Reemergence of rheumatic fever after a systemic streptococcal A infection: The role of cardiac MRI on the diagnosis of subclinical rheumatic carditis
}

\author{
Authors: John Serelis, ${ }^{A}$ Nikolaos Alexopoulos, ${ }^{B}$ Fotini N Skopouli ${ }^{C}$ and Haralampos M Moutsopoulos ${ }^{D}$
}

\begin{abstract}
A 42-year-old man presented with fever, sore throat, rash and painful right knee swelling, preceded by self-medication with oral steroids. Blood and knee cultures yielded group A Streptococcus. After 2 weeks of intravenous antibiotics and two arthroscopic knee debridements, he continued to experience spiking fevers, and electrocardiographic changes developed. We postulate that the patient suffered from the first presentation of acute rheumatic fever, following an invasive group A bacteraemic streptococcal infection. The possible role of cardiac magnetic resonance imaging in the diagnosis of rheumatic carditis is discussed.
\end{abstract}

KEYWORDS: Bacteraemia, septic arthritis, Group A streptococcus, acute rheumatic fever, cardiac MRI

DOI: $10.7861 /$ clinmed.2020-0163

\section{Case presentation}

A 42-year-old man came to the emergency department with fever, generalised weakness as well as painful and oedematous right knee and right elbow. He had a history of recurrent attacks of oedematous swelling of the uvula of undetermined etiology, for which he was self-medicated with a 3-day course of oral methylprednisolone. Two days after the last steroid course he was admitted with spiking fevers $\left(38.5-39^{\circ} \mathrm{C}\right)$ associated with painful knee and right elbow.

The patient's 3-year-old son, a few days prior to the patient's symptoms, had a sore throat. The patient's past and family history was noncontributory except that his father had died at the age of 45 years from heart attack.

Upon admission he was febrile (axillary temperature of $39.2^{\circ} \mathrm{C}$ ), tachycardic (pulse rate of 108 beats/minute) and appeared to be in considerable distress. An erythematous plaque (diameter of $2 \mathrm{~cm}$ ) was noted on his nose (Fig 1a), as well as erythematous,

Authors: ${ }^{\text {A }}$ consultant physician, Athens Euroclinic, Athens, Greece; ${ }^{B}$ head cardiologist, Athens Euroclinic, Athens, Greece; ${ }^{C}$ director and department head, Athens Euroclinic, Athens, Greece; ${ }^{D}$ chair of medical sciences-immunology, Athens Academy, Athens, Greece swollen, painful right knee and right elbow. Laboratory tests showed leukocytosis; white blood cell count (WBC) was $20.3 \times 10^{9} / \mathrm{L}$ and elevated C-reactive protein (CRP) at $25.5 \mathrm{mg} / \mathrm{L}(<0.5 \mathrm{mg} / \mathrm{L})$. Arthrocentesis of the knee yielded a purulent synovial fluid containing $18.5 \times 10^{9} / \mathrm{L}$ white cells ( $80 \%$ polymorphonuclear leukocytes), increased protein at $4.28 \mathrm{~g} / \mathrm{dL}$, decreased glucose levels at $2.1 \mathrm{mmol} / \mathrm{L}$ and was negative for crystals. The valves and ejection fraction were normal on echocardiography at admission. With presumed diagnosis of septic arthritis, the patient was initiated on intravenous administration of ceftriaxone $2 \mathrm{~g}$ twice daily. Blood and synovial fluid cultures grew group A Streptococcus. Intravenous clindamycin at 900 mg every 8 hours was added. The knee was aspirated and drained arthroscopically. The facial erythematous plaque and the elbow arthritis had subsided considerably the day after the initiation of antibiotic therapy. However, despite the patient's condition improving gradually, he continued to experience daily spiking fevers and right knee swelling. A second surgical drainage was performed on day 15 of admission. Blood, throat and synovial fluid cultures were repeatedly negative while the CRP levels remained moderately elevated ( $6 \mathrm{mg} / \mathrm{L})$. Twenty days after his presentation, the serum anti-streptolysin $\mathrm{O}$ (ASTO) levels were highly elevated at 1,909 IU/mL. At that time, electrocardiography (ECG) revealed inverted T waves (on the inferior II, III and aVF, and lateral leads V5 and V6), which were not present on admission or on day 15, while cardiac troponin-T levels were within normal limits (Fig 1b). Repeated echocardiography was normal. Cardiac magnetic resonance imaging (MRI) showed normal valves, ventricular volumes and systolic function. There was, however, increased signal intensity in T2-weighted (STIR) sequences and in late-gadolinium enhancement images in the sub-epicardial layer of the basal-inferior, basal-inferolateral and apical lateral left ventricular wall (Fig 2), findings compatible with acute focal myocarditis. The patient was placed on angiotensinconverting enzyme inhibitor and small doses of beta blocker. The diagnosis of acute rheumatic fever (ARF) was placed since the clinical picture consisting of fever, arthritis and carditis followed a streptococcal group A infection. The patient was started on naproxen 500 mg twice daily and intramuscular injection of benzathine penicillin monthly. He was re-evaluated 15 days after discharge and complete remission of the clinical and serological picture was observed, while 1 month later the electrocardiographic changes had also abated. 
Fig 1. a) Photograph of an erythematous plaque (diameter of $2 \mathrm{~cm}$ ) on the nose. b) Electrocardiography revealing inverted T waves (on the inferior II, III and $\mathrm{aVF}$, and lateral leads V5 and V6).
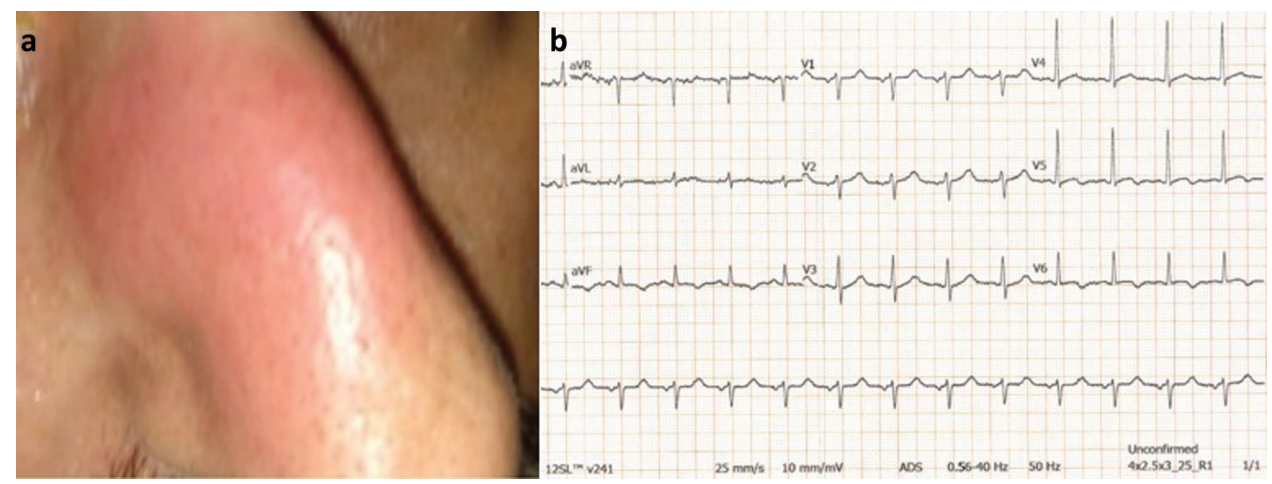

\section{Discussion}

Streptococcus group A is a ubiquitous Gram-positive bacterium which can produce a range of different clinical manifestations from local disease (usually pharyngitis) to systemic bacteraemia, with or without localised infection (arthritis, meningitis or pneumonia) or septic shock syndrome to non-suppurative sequelae such as post-streptococcal glomerulonephritis and rheumatic fever. ${ }^{1}$ We present this case since almost all the streptococcal clinical disease spectrum was expressed in a short period of time, from mistreated pharyngitis bacteraemia developed, by localised streptococcal manifestations (skin lesions and septic arthritis). Finally, ARF developed, expressed by persistent fever, severe aseptic knee synovitis, rheumatic carditis and serum inflammatory indices. The development of subclinical carditis 20 days after the initial manifestations, while the infection was under control, is significant since it led us to initiate the appropriate anti-inflammatory therapy as well as the administration of monthly intramuscular penicillin for secondary prevention.

ARF typically manifests 2 weeks to several months after a throat infection. The diagnosis was based on modified Jones criteria including major, minor and supportive criteria. ${ }^{2,3}$ Our patient had two major criteria (arthritis and carditis) and two minor (fever and elevated (RP) as well as supportive criteria (Streptococcus
A positive cultures, elevated serum ASTO levels). Since carditis developed in a patient with bacteraemia, it might be argued that the myocardial involvement was the result of the streptococcal bacteraemia itself. However, the clinical/serologic picture of acute streptococcal non-rheumatic myocarditis is not common, presenting usually with chest pain, ST segment elevation on ECG, elevated cardiac enzymes and impaired ejection fraction, mimicking ST-elevation myocardial infarction (STEMI) in young adults. ${ }^{4,5,6}$ Furthermore, streptococcal myocarditis is manifested 3-4 days after pharyngitis. ${ }^{6}$ Thus, the possibility that our patient suffered from an acute non-rheumatic streptococcal myocarditis is remote. Generally, rheumatic carditis has been considered as a pancarditis involving the heart valves, myocardium and pericardium. Subclinical carditis may express itself with aortic or mitral regurgitation on Doppler echocardiography in patients without any clinical evidence of carditis. In this case, using cardiac MRI, we detected the inflammatory lesion just below epicardium, which is consistent with the diagnosis of acute focal myocarditis. Another case of isolated myocarditis in adult-onset

ARF diagnosed and followed-up by cardiac MRI has been reported, while a paediatric ARF case presenting with complete atrioventricular block attributed to focal myocarditis which was also identified by a heart MRI was described in Japan.,

In conclusion, ARF can still be seen when streptococcal pharyngitis is mistreated. Subclinical focal myocarditis with

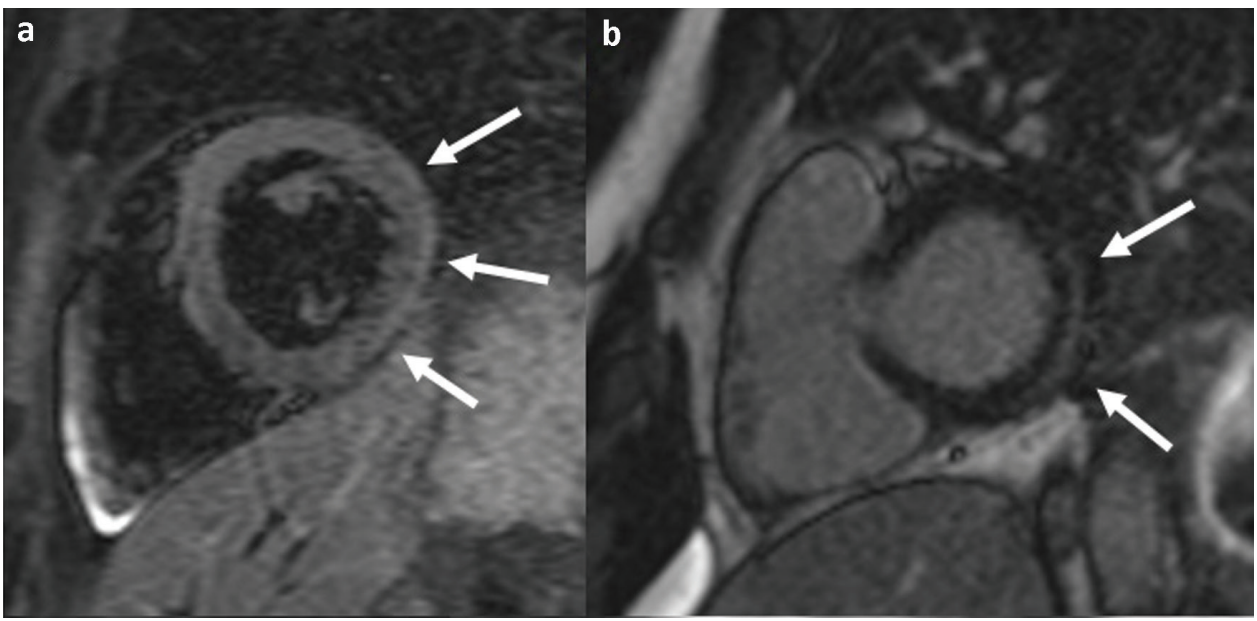

Fig 2. a) Increased signal intensity in T2-weighted (STIR) magnetic resonance imaging sequence and (b) late-gadolinium enhancement imaging of the sub-epicardial layer of the basalinferior, basal-inferolateral and apical lateral left ventricular wall (arrows), findings are compatible with acute focal myocarditis. 
inverted T waves, normal contractility and cardiac enzymes should not be overlooked and should be considered as a potential ARF manifestation, with cardiac MRI presenting as a useful diagnostic tool.

\section{References}

1 Carapetis JR, Steer AC, Mulholland EK, Weber M. The global burden of group A streptococcal diseases. Lancet Infect Dis 2005;5:68594.

2 Gewitz MH, Baltimore RS, Tani LY et al. Revision of the Jones Criteria for the diagnosis of acute rheumatic fever in the era of Doppler echocardiography: a scientific statement from the American Heart Association. Circulation 2015;131:1806-18.

3 Rammelkamp CH, Stolzer BL. The latent period before the onset of acute rheumatic fever. Yale J Biol Med 1961;34:386-98.

4 Gill MV, Klein NC, Cunha BA. Nonrheumatic poststreptococcal myocarditis. Heart Lung 1995;24:425-6.
5 Upadhyay GA, Gainor JF, Stamm LM et al. Acute nonrheumatic streptococcal myocarditis: STEMI mimic in young adults. Am J Med 2012:125:1230-3.

6 Derbas LA, Samanta A, Potla S et al. Separating acute rheumatic fever from nonrheumatic streptococcal myocarditis. Case Rep Med 2019:2019:4674875.

7 Moriwaki K, Okamoto R, Sakuma H et al. Tracking isolated myocarditis in a young adult with acute rheumatic fever on cardiac magnetic resonance imaging. Int J Cardiol 2016;222:921-3.

8 Kunimatsu M, Ebata R, Takaoka H et al. Focal myocarditis with complete atrioventricular block as an initial presentation of rheumatic fever. Chiba Med J 2017;93E:59-62.

Address for correspondence: Dr John Serelis, Department of Medicine and Clinical Immunology, Athens Euroclinic, 7-9 Athanasiadou Street, Athens 11521, Greece.

Email: iserelis@hotmail.com 